\title{
Tuning localized plasmon cavities for optimized surface-enhanced Raman scattering
}

\author{
N. M. B. Perney, ${ }^{1, *}$ F. J. García de Abajo, ${ }^{2}$ J. J. Baumberg, ${ }^{1}$ A. Tang, ${ }^{3}$ M. C. Netti, ${ }^{3}$ M. D. B. Charlton, ${ }^{3}$ and M. E. Zoorob ${ }^{3}$ \\ ${ }^{1}$ School of Physics and Astronomy, University of Southampton, Highfield, Southampton SO17 1BJ, United Kingdom \\ ${ }^{2}$ Instituto de Optica-CSIC, Serrano 121, 28006 Madrid, Spain \\ ${ }^{3}$ Mesophotonics Ltd., 2 Venture Road, Southampton SO16 7NP, United Kingdom
}

(Received 13 October 2006; published 20 July 2007)

\begin{abstract}
Mesostructured metallic substrates composed of square pyramidal pits are shown to confine localized plasmons. Plasmon frequency tuning is demonstrated using white light reflection spectroscopy with a wide range of structure dimensions from 400 to $3000 \mathrm{~nm}$. Using a simple plasmon cavity model, we demonstrate how the individual pit morphology and not their periodicity controls the resonance frequencies. By measuring the surface-enhanced Raman scattering (SERS) signals from monolayers of benzenethiol on the same range of mesostructures, we extract a quantitative connection between absorption, field enhancement, and SERS signals. The match between theory and experiment enables effective design of plasmon devices tailored for particular applications, such as optimizing SERS substrates.
\end{abstract}

DOI: 10.1103/PhysRevB.76.035426

PACS number(s): 73.20.Mf, 42.70.Qs, 72.15.Rn, 81.07.-b

Raman scattering is a crucial spectroscopic technique for identifying molecules through their vibrational resonances and has increasingly important applications in monitoring low concentrations of impurities or trace biomolecules. ${ }^{1-3}$ It has also been suggested for direct monitoring of coupling of molecular distortions and electronic transport in molecular electronics. ${ }^{4-6}$ However, the terribly weak Raman cross section has always made such application problematic. The enormous enhancement in cross section when the molecules are held close to a metal surface with nanoscale roughness ${ }^{7,8}$ has driven the hope that surface-enhanced Raman scattering (SERS) will become a viable and reproducible diagnostic. While improvements have been made in terms of enhancement factor, reproducibility, and in understanding that plasmons underpin such enhancements, ${ }^{9}$ it is unclear how to precisely design nanostructures to optimize the Raman signatures. Over the last five years, we have shown that mesostructured metal films comprised of arrays of voids form excellent surfaces for localizing plasmons while retaining strong coupling to external light. ${ }^{10-13}$ Recently, we showed, using angularly resolved SERS on such void substrates, that incident photons are transducted both into and out of molecules via plasmons, ${ }^{14}$ giving hope that reproducible substrates can be designed for specific applications. While most SERS research has focused on using the nanoscale junctions between metallic particles such as colloids ${ }^{15}$ or lithographic arrays $^{16}$ that have broad plasmon resonances which can only be tuned through control of shape anisotropy or gap dimensions, the voids show strong sharp tunable plasmon resonances. Previous work has shown that optimized samples possess plasmon absorption that lies between the laser wavelength and outscattered Raman emission; ${ }^{17-19}$ however, they are unable to make clear the quantitative link between plasmon resonant absorption and SERS emission.

In this paper, we present calibrated spectroscopic measurements on systematically engineered plasmonic mesostructured metal surfaces. We show the quantitative connection between the resonance arising from wavelength scale gold mesostructures and the SERS enhancement of a monolayer of molecules. The metallic substrates are composed of arrays of inverted pyramidal pits which we have recently developed ${ }^{20}$ to provide extremely accurate control and reproducibility over the void morphology. In complete contrast to metallodielectric plasmonic crystals, ${ }^{21}$ we show that such voids have plasmonic resonances controlled only by the geometry of individual voids. We are able to account for this behavior using a simple plasmon cavity model, which makes quantitative predictions about the SERS enhancement. The excellent agreement between theory and experiment allows straightforward SERS substrate design for specific applications.

We fabricate a matrix of samples from (100) oriented silicon wafers, with square pit apertures aligned along the [110] directions. $^{22}$ Arrays of apertures defined by electron-beam lithography are transferred into a $\mathrm{Cr}$ metal mask. An anisotropic $\mathrm{KOH}$ etch is used to preferentially etch to the $\{111\}$ planes forming an array of inverted pyramidal pits, as seen in Fig. 1. This fabrication technique is instrumental in producing extremely reproducible samples, with a fixed apex pit angle of $\arccos (1 / 3)=70.5^{\circ}$, the surfaces of which are atomically smooth. ${ }^{22}$ This is crucial in order to develop a detailed understanding of the correlation between plasmon absorption and SERS, which has been complicated by problems in achieving plasmon tuning while maintaining the nanoscale landscape. ${ }^{23}$ Previously, we showed that small changes in the pit dimensions modified the plasmon resonant frequency. ${ }^{13}$ Here, we map the entire plasmon landscape, moving between regimes where the incident optical wavelength is both much larger and much smaller than the void feature size. In order
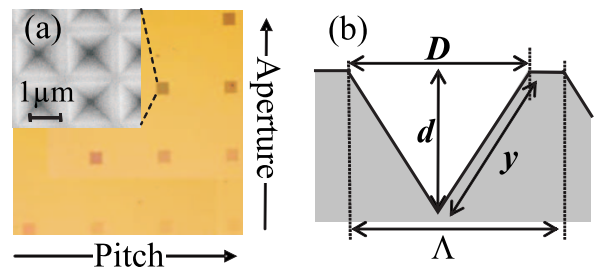

FIG. 1. (Color online) (a) Optical micrograph of a gold pyramidal pit array showing $50 \mu \mathrm{m}$ patches with pitch and aperture graded as indicated across the sample. Inset: SEM of several pits. (b) Cross section of pit parallel to edge. 


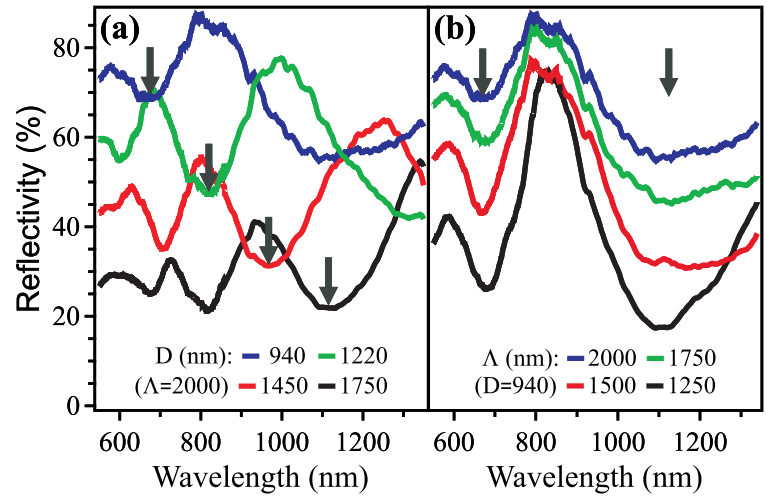

FIG. 2. (Color online) Reflectivity spectra of pit arrays with varying (a) aperture size and (b) pitch. The arrows indicate the position of resonant absorption features.

to distinguish localized plasmon and diffraction effects, we vary the aperture size $D$ from $300 \mathrm{~nm}$ to $2.0 \mu \mathrm{m}$ and the pitch $\Lambda$ from $500 \mathrm{~nm}$ to $3 \mu \mathrm{m}$, with each $(D, \Lambda)$ combination realized in a $50 \mu \mathrm{m}^{2}$ square. The samples were then coated by sputtering with $300 \mathrm{~nm}$ of gold and inspected by FEG-SEM to confirm that the metal texture inside the pits and on the flat top is identical, showing typical sub-50-nm scale roughness. We have confirmed that the precise $\mathrm{Au}$ thickness makes little difference to the plasmon resonances observed and also that other noble metals give very similar results. $^{24}$

We have previously shown through angle-resolved whitelight-laser spectroscopy that the localized plasmon absorption bands are independent of the angle of incidence but are modulated by angle-dependent diffraction features. ${ }^{13}$ Here, our main goal is to correlate the plasmon absorption and the measured SERS; hence, both are measured using a microscope objective at normal incidence which integrates over a range of angles and averages out diffraction features. Reflectivity spectra are recorded with unpolarized white light through a $20 \times$ microscope objective, numerical aperture of 0.4 , using visible and infrared spectrometers. As expected, restricting the angular aperture to vary the range of incident angles from $\pm 23^{\circ}$ down to $\pm 5^{\circ}$ has no effect on the spectra. Spectra are recorded and normalized against a flat gold section of the same sample, thereby eliminating optical effects due to the metal coating. The SERS spectra are recorded using a commercial Raman system, with $785 \mathrm{~nm}$ pump laser of $10 \mathrm{~mW}$ power, with $10 \mathrm{~s}$ integration time and $20 \times$ microscope objective.

Clear evidence of plasmon tuning is presented in Fig. 2(a) which shows spectra for a fixed pitch of $2000 \mathrm{~nm}$ and aperture varying from 940 to $1750 \mathrm{~nm}$. The resonant absorption features (one of which is indicated by arrows) shift to longer wavelength with increasing pit aperture. By contrast, varying the pitch at constant aperture size has no influence on the resonance [Fig. 2(b)], which is instead only sensitive to the number of pits per unit area. We note that this is completely different from metallodielectric plasmonic crystals as the modes probed here are specifically the localized plasmons. The reflectivity spectra overlap in magnitude when normalized to the pit filling fraction, allowing us to confirm that the plasmons in the pits are isolated from each other.

The resonant plasmon wavelength depends only on the geometry of the individual pits. We first confirm that the tuning of the plasmons follows the simple surface-plasmonpolariton standing wave model that we introduced recently (but only over a very limited parameter space). ${ }^{13}$ This model assumes that plasmons oscillate backward and forward up and down the sides of each pit, completely reflected by the top convex sharp pit edges. Plasmon interference implies that only certain plasmon energies fit within the $2 y$ path length, leading to a round trip phase $\phi=k_{s p p} \times 2 y=4 \pi n_{s p p} y / \lambda$. Extracting the experimental reflectivity dips and plotting their energy as a function of aperture size [Fig. 3(a)] show a surprisingly good match with theory (dashed lines), supporting the proposed model. The energy separation of the modes (controlled by the effective plasmon cavity length) only fits well for plasmons confined to the surface of the pit walls (rather than, for instance, across the aperture). For the larger structures, up to 6 orders of plasmon standing waves are visible, equivalent to the higher plasmon modes recently observed in nanorods. ${ }^{25}$

To extend this model, we discuss the metal void in terms of a resonant plasmon cavity [Fig. 3(b)]. The field coupling strength of incoming light into the plasmon cavity is $t$, with $r$ directly reflected and the fraction of plasmon field not absorbed in the cavity on each round trip is $a$, with the round trip phase shift of $\phi$ as above. Evaluating the multiply reflected plasmon field, the light absorbed from the field in the cavity is $E_{t o t} \times \sqrt{1-a^{2}}$, giving for the absorption

$$
\alpha=\frac{\left(1-r^{2}\right)\left(1-a^{2}\right)}{\left|1-r a e^{-i \phi}\right|^{2}} \text {. }
$$

Extracting the absorption linewidth in radians ( $\Gamma$ is the half-width at half maximum) and the maximum absorption $\left(\alpha_{m}\right)$ from Eq. (1) and inverting the resulting equations give expressions for the cavity parameters in terms of the measured absorption spectra,

$$
\begin{aligned}
& a^{2}=\exp \{-\gamma+\beta\}, \\
& r^{2}=\exp \{-\gamma-\beta\},
\end{aligned}
$$

where $\cosh \gamma=2-\cos \Gamma$ and $\cosh \beta=\alpha_{m}+\cosh \gamma\left(1-\alpha_{m}\right)$. Hence, by measuring $\alpha_{m}$ and $\Gamma$, we find $a$ and $r$ and hence evaluate the field enhancement at each wavelength from

$$
E^{2}(\lambda)=\left|\frac{\varepsilon_{\text {cavity }}(\lambda)}{\varepsilon_{\text {incident }}}\right|^{2}=\frac{\alpha(\lambda)}{1-a^{2}} .
$$

In order to extract the plasmonic absorption, we normalize the reflection spectra to account for the nonplasmonic scattering. For light whose wavelength is much smaller than the pit features, a ray optics model shows that all light incident on the pit is reflected at angles not collected by our optical configuration. ${ }^{22}$ Thus, the reflectivity is set by the fill fraction of pits in the reflection upper surface. On the other hand, for light whose wavelength is much larger than the pit features, an effective refractive index for the surface combined with pit-induced scattering into all directions is obtained. Combining these models allows effective fitting of 

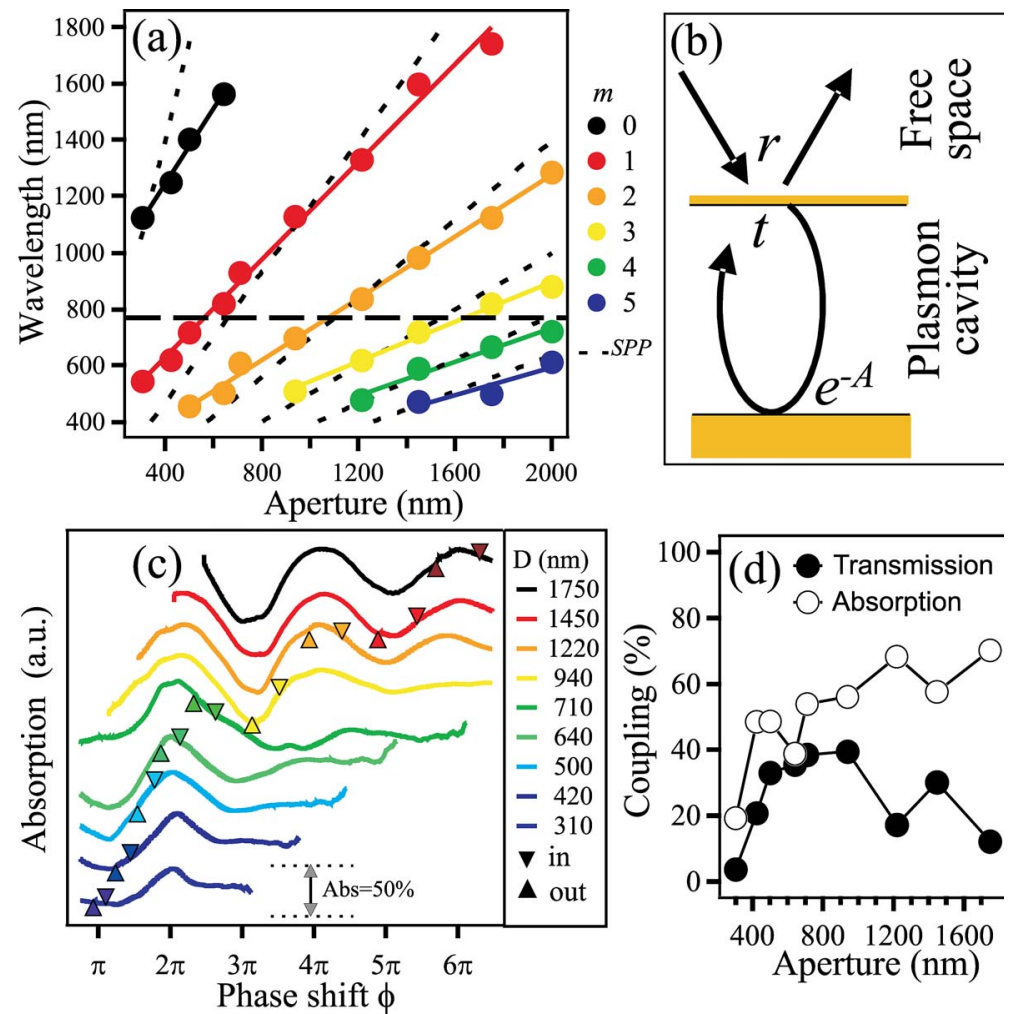

FIG. 3. (Color online) (a) Experimental plasmon resonant wavelengths vs aperture size, with theory (dashed lines) and horizontal long dashed line at the ingoing laser wavelength. (b) Schematic plasmon cavity. (c) Absorption spectra, $\alpha$ $=1-R$ (shifted for clarity), plotted against the cavity phase shift. The triangles correspond to the laser excitation (down) and Raman shifted (up) wavelengths. (d) Extracted transmission and absorption coefficients of the cavity as a function of aperture size. the background reflectivity, as confirmed by measurements on a large number of structured metals and dielectrics. ${ }^{24}$

The resulting absorption spectra for different pit dimensions are plotted against the round trip phase shift [Fig. 3(c)], showing the expected resonance condition every $2 \pi$, consistent with cavity behavior. From this, the absorption linewidth and amplitude can be extracted as well as the phase shift between the absorption peaks. From such fits, the coupling strength is found to peak at $40 \%$, decreasing for either very small or large cavities [Fig. 3(d)]. On the other hand, the maximum absorption per round trip $(1-a)$ increases from $20 \%$ to $70 \%$ as the cavity size increases [Fig. 3(d)]. This can be understood intuitively from the increasing lossy sidewall length in larger cavities: we find a scaling equivalent to $-1 \mathrm{~dB} / \mu \mathrm{m}$, with an additional fixed contribution of $1 \mathrm{~dB}$ (which could be ascribed to scattering at the pit apex). This should be compared with surface plasmon-polaritons on a flat $\mathrm{Au}$ surface at the same wavelengths, which have eight times less dissipation.

Using Eq. (4) provides an estimate of the field strength as a function of wavelength for each cavity. This has been thought to be the crucial parameter for SERS ${ }^{18}$ but has not previously been quantitatively controlled in plasmonic mesostructures. The Raman scattered emission at $\omega_{p}-\nu_{i}$ when pumping $\left(\right.$ at $\left.\omega_{p}\right)$ the molecules with $i$ th vibrational resonance at $\nu_{i}$ is

$$
I_{S E R S} \propto E^{2}\left(\omega_{p}\right) E^{2}\left(\omega_{p}-\nu_{i}\right) .
$$

From the spectra in Fig. 3(c), we can thus predict the SERS expected from each of the structured voids. To provide a good quantitative SERS comparison, we use a monolayer molecular marker allowing calibrated measurement of the Raman cross section. The plasmon substrate samples are immersed for $8 \mathrm{~h}$ in a solution of benzenethiol in ethanol and the excess subsequently washed off with ethanol. This procedure ensures monolayer coverage of the surface of the sample by the molecules. ${ }^{14,26}$ We have previously demonstrated that these mesostructured substrates have a SERS reproducibility and uniformity below $10 \%$ under these conditions. No SERS signal could be detected on the flat portion of the sample, indicating that the surface is sufficiently smooth not to provide nanoscale field localization (hot spots). From SERS spectra of each patch [Fig. 4(a)], the intensity of the strong $1070 \mathrm{~cm}^{-1}$ line is extracted. What is also apparent in this data is that the enhancement is similar for all the vibrational lines and also for the background (whose origin remains unclear). To simplify the analysis and effects of fill fraction, we concentrate the analysis on the
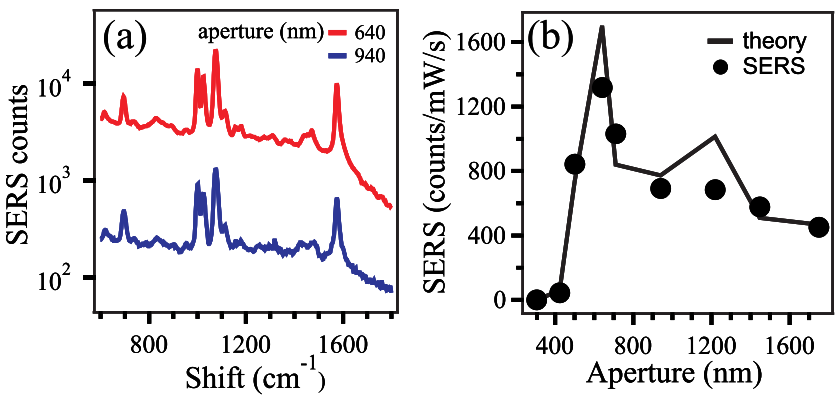

FIG. 4. (Color online) (a) SERS spectra for two different pit apertures, extracted peak height shown as dashed line. (b) SERS counts normalized for exposure and incident power and predicted SERS enhancement from Eq. (5) as a function of aperture size. 
arrays that have the maximum filling fraction of pits of around $70 \%$ and therefore constant pit wall area. The observed SERS counts are normalized for laser power and acquisition time. This normalized SERS shows a strong dependence on the pit dimensions, varying by $>10^{4}$ and exhibiting two strong maxima at apertures of 640 and $940 \mathrm{~nm}$. We measured four different samples and found the same systematic trend to be repeatable; the average is shown in Fig. 4(b). These SERS resonances correspond to situations when pump and emitted Raman photons are in resonance with the localized plasmons, as seen for the dashed line at the pump photon energy in Fig. 3(a).

Plotting the predicted and measured SERS enhancements vs aperture size [Fig. 4(b)] shows the excellent agreement and supports the validity of using our highly simplified plasmon cavity model. The situation for both ingoing and outgoing resonances can be examined in more detail in Fig. 3(c), where the corresponding energetic positions are marked (in terms of the phase shift). The strongest SERS enhancement is seen for the smallest pits which enclose a single plasmon wavelength and display the strongest plasmon absorption. Higher order plasmon resonances (such as $m=3$ ) are nearly ten times less SERS active. These features also clearly demonstrate that such plasmon mesostructured substrates cannot be used for all applications at all laser wavelengths. Instead, it is necessary to appropriately match the void geometry to the pump laser wavelength.

Several features remain to be fully understood in such plasmon nanovoids. Clearly, the input coupling and the round trip absorption change with void geometry; however, the full field distribution in such structures and the way that incoming plane waves couple to them remain less clear. Recently, we have managed to model the full three-dimensional field using a boundary element method when the pits are approximated as inverted cones. These calculations broadly confirm the plasmon standing wave model, with the electric field position depending on the size of the pit. ${ }^{27}$ These calculations also confirm that the void geometry shows much stronger field enhancements than flat gold squares of equivalent area, as the plasmons are drawn down into the pits, better confined and sucked into contact with the metal surface. The strong absorption is indicative of the strength of the electric field on the metal surface, which is critical for SERS enhancements. ${ }^{28}$

In conclusion, gold coated submicron pits are shown to behave as plasmon cavities, with field enhancements controlled by the coupling and intracavity plasmon absorption. For the pyramidal pits used here, their rather open structure allows for good physical access so the molecules to be sensed easily reach the metallic surface. However, this restricts the quality factors which are attainable. In a similar way to transmission through subwavelength aperture holes, ${ }^{29}$ the plasmon modes depend on the material dielectric properties of the pit. Moreover, the tapered geometry of the inverted pits is influential in reducing reflection and increasing input coupling. ${ }^{30}$ Although diffractive coupling can couple propagating plasmons onto the top metal surface, the absence of any dependence on the pitch of our arrays suggests that this mechanism does not strongly contribute to plasmon coupling and hence SERS enhancements.

Understanding the localized plasmon modes responsible for the field enhancement in nanostructured surfaces is the first step toward controlled SERS substrates. Control of the coupling mechanism needs to be understood so as to efficiently channel radiation in and out of the localized modes. By interpreting the inverted pits as resonant plasmon cavities, we model both field enhancement and coupling and show clear correlation between measured absorption spectra and SERS. This work suggests that many types of metallic mesostructures can be helpfully treated as plasmonic cavities.

This work was supported by EPSRC NanoPhotonics Portfolio EP/C511786/1. We would like to gratefully acknowledge helpful discussions with F. Birembaut, Y. Sugawara, P. N. Bartlett, M. Abdelsalam, and A. E. Russell. *nmbp@phys.soton.ac.uk

${ }^{1}$ F. Bertram, T. Riemann, J. Christen, A. Kaschner, A. Hoffmann, C. Thomsen, K. Hiramatsu, and N. Sawaki, Appl. Phys. Lett. 74, 359 (1999).

${ }^{2}$ R. Schweitzer-Stenner, J. Raman Spectrosc. 36, 276 (2005).

${ }^{3}$ R. C. Lord, Appl. Spectrosc. 31, 187 (1977).

${ }^{4}$ M. A. Rohrdanz and J. A. Cina, Mol. Phys. 104, 1161 (2006).

${ }^{5}$ A. Jaiswal, K. G. Tavakoli, and S. Zou, Anal. Chem. 78, 120 (2006).

${ }^{6}$ A. M. Nowak and R. L. McCreery, Anal. Chem. 76, 1089 (2004).

${ }^{7}$ M. Fleischman, P. J. Hendra, and A. J. McQuillan, Chem. Phys. Lett. 26, 163 (1974).

${ }^{8}$ D. L. Jeanmaire and R. P. Van Duyne, J. Electroanal. Chem. 84, 1 (1977).

${ }^{9}$ K. Kneipp, H. Kneipp, I. Itzkan, R. R. Dasari, and M. S. Feld, J. Phys.: Condens. Matter 14, R597 (2002).

${ }^{10}$ T. A. Kelf, Y. Sugawara, J. J. Baumberg, M. Abdelsalam, and P.
N. Bartlett, Phys. Rev. Lett. 95, 116802 (2005).

${ }^{11}$ S. Coyle, M. C. Netti, J. J. Baumberg, M. A. Ghanem, P. R. Birkin, P. N. Bartlett, and D. M. Whittaker, Phys. Rev. Lett. 87, 176801 (2001).

${ }^{12}$ J. Aizpurua, P. Hanarp, D. S. Sutherland, M. Kall, Garnett W. Bryant, and F. J. Garcia de Abajo, Phys. Rev. Lett. 90, 057401 (2003).

${ }^{13}$ N. M. B. Perney, J. J. Baumberg, M. E. Zoorob, M. D. B. Charlton, S. Mahnkopf, and M. C. Netti, Opt. Express 14, 847 (2006).

${ }^{14}$ J. J. Baumberg, T. A. Kelf, Y. Sugawara, S. Cintra, M. E. Abdelsalam, P. N. Bartlett, and A. E. Russell, Nano Lett. 5, 2262 (2005).

${ }^{15}$ K. Kneipp, A. S. Haka, H. Kneipp, K. Badizadegan, N. Yoshizawa, C. Boone, K. E. Shafer-Peltier, J. T. Motz, R. R. Dasari, and M. S. Feld, Appl. Spectrosc. 56, 150 (2002).

${ }^{16}$ C. L. Haynes, A. D. McFarland, and R. P. Van Duyne, Anal. 
Chem. 77, 338A (2005).

${ }^{17}$ A. D. McFarland, M. A. Young, J. A. Dieringer, and R. P. Van Duyne, J. Phys. Chem. B 109, 11279 (2005).

${ }^{18}$ N. Félidj, J. Aubard, and G. Lévi, Appl. Phys. Lett. 82, 3095 (2003)

${ }^{19}$ J. Grand, S. Kostcheev, J.-L. Bijeon, M. Lamy de la Chapelle, P.-M. Adam, A. Rumyantseva, G. Lérondel, and P. Royer, Synth. Met. 139, 621 (2003).

${ }^{20} \mathrm{http} / / / \mathrm{www} . \mathrm{mesophotonics.com/products/klarite.html}$

${ }^{21}$ A. Christ, S. G. Tikhodeev, N. A. Gippius, J. Kuhl, and H. Giessen, Phys. Rev. Lett. 91, 183901 (2003).

${ }^{22}$ M. Trupke, F. Ramirez-Martinez, E. A. Curtis, J. P. Ashmore, S. Eriksson, E. A. Hinds, Z. Moktadir, C. Gollasch, M. Kraft, G. V. Prakash, and J. J. Baumberg, Appl. Phys. Lett. 88, 071116 (2006).
${ }^{23}$ J. A. Dieringer, O. Lyandres, A. D. McFarland, N. C. Shah, D. A. Stuart, A. V. Whitney, C. R. Yonzon, M. A. Young, J. Yuen, X. Zhang, and R. P. Van Duyne, Faraday Discuss. 132, 9 (2006).

${ }^{24}$ N. M. B. Perney et al. (unpublished).

${ }^{25}$ G. Laurent, N. Félidj, J. Aubard, G. Lévi, J. R. Krenn, A. Hohenau, G. Schider, A. Leitner, and F. R. Aussenegg, J. Chem. Phys. 122, 011102 (2005).

${ }^{26}$ S. Cintra, M. E. Abdelsalam, P. N. Bartlett, J. J. Baumberg, T. A. Kelf, Y. Sugawara, and A. E. Russell, Faraday Discuss. 136, 16 (2005).

${ }^{27}$ J. Garcia de Abajo et al. (unpublished).

${ }^{28}$ S. I. Bozhevolnyi, V. S. Volkov, E. Devaux, J.-Y. Laluet, and T. W. Ebbesen, Nature (London) 440, 508 (2006).

${ }^{29}$ H. J. Lezec and T. Thio, Opt. Express 12, 3629 (2004).

${ }^{30}$ P. Cambell and M. A. Green, J. Appl. Phys. 62, 243 (1987). 